\title{
Chronic Sinusitis
}

National Cancer Institute

\section{Source}

National Cancer Institute. Chronic Sinusitis. NCI Thesaurus. Code C35151.

Inflammation of the paranasal sinuses that typically lasts beyond eight weeks. It is caused by infections, allergies, and the presence of sinus polyps or deviated septum. Signs and symptoms include headache, nasal discharge, swelling in the face, dizziness, and breathing difficulties. 